\title{
The use of tracheal stoma stents in high spinal cord injury: a patient-friendly alternative to long-term tracheostomy tubes
}

\author{
AM Hall and JWH Watt \\ North West Regional Spinal Injuries Centre, Southport and Formby District General Hospital, Southport, UK
}

\begin{abstract}
Study design: Case series.
Setting: North West Regional Spinal Injuries Unit, Southport and Formby District General Hospital, UK.

Objectives: To identify a novel type of tracheal stents for use in patients with high spinal cord injury. Patients with high spinal cord injury (above C4) frequently have significant respiratory impairment and may require long-term access to the trachea for respiratory support. For the most part, tracheostomy tubes are used for this purpose but a tracheal stoma stent can offer a suitable alternative in selected cases and deserves wider recognition.

Methods: Following completion of a patient questionnaire survey, the authors describe the use of stoma stents in nine patients, five of whom had full-time diaphragm pacing. The stent in these cases is for retention of access for positive pressure ventilation, and for the prevention of obstructive sleep apnoea. This was also the indication in one self-ventilating patient with tetraplegia and sleep apnoea. Two patients with recurrent chest infections, in whom chest physiotherapy was difficult, benefited from the stoma stents. One patient, after ventilator weaning, required a further 4 months of tracheal access on account of episodic hypoventilation and temporarily had a tracheal stent as an inpatient.

Conclusion: Patients who have had the benefit of tracheal stents report significant improvement in relation to local discomfort, tracheobronchial secretions and vocalization. With suitable training, the stents can be changed and cleaned easily in the home setting.

Spinal Cord (2008) 46, 753-755; doi:10.1038/sc.2008.18; published online 18 March 2008
\end{abstract}

Keywords: high spinal cord injury; tracheal stoma stents; long-term ventilation

\section{Introduction}

Patients with high spinal cord injury (above C4) have inspiratory muscle weakness and may need long-term respiratory support, usually through a tracheostomy. The need depends on the extent of involvement of the phrenic motoneurones. In patients with upper motorneurone phrenic nerve paralysis, phrenic-nerve-pacing devices may be used for respiratory assistance. Inframedullary stimulation of the diaphragm in this way typically provokes obstructed breathing during sleep because the respiratory centre fails to generate the necessary phasic and tonic genioglossus contraction. It is, therefore, advisable to retain the tracheostomy to relieve obstructed breathing, to maintain access for tracheal toilet and for emergency access for mechanical ventilation. $^{1}$

Correspondence: Dr AM Hall, Department of Anaesthesia, Southport and Formby District General Hospital, Town Lane Kew, Southport, Merseyside PR8 6PN, UK.

E-mail: alih101@yahoo.com

Received 21 November 2007; revised 15 January 2008; accepted 31 January 2008; published online 18 March 2008
Even tetraplegic patients with fully preserved diaphragm function have respiratory vulnerability due to profound expiratory weakness. While assisted coughing is helpful for clearing tracheobronchial secretions, this may not be adequate in patients with rigid chest wall or poor coordination.

A tracheostomy tube, cuffed or uncuffed, is the definitive airway for the purpose of providing positive pressure ventilation. They are also widely used to facilitate directed suctioning into the lower trachea. They do, however, have several disadvantages including pain, discomfort, tracheal stenosis and ischaemic necrosis. In addition, the cosmetic appearance of these tubes can be psychologically disturbing.

The presence of the tracheostomy tube in these patients may in itself engender tracheobronchial secretions and impede their natural clearance so, as an alternative, a tracheal stoma stent could be considered. Believing that this approach could be used more widely, the authors have reviewed the recent experience at the North West Regional Spinal Injuries Centre (NWRSIC). 


\section{Case series}

The authors describe the use of stoma stents in nine patients over the past decade, five of whom had full-time diaphragm pacing due to injury above C3.

One self-ventilating patient with tetraplegia and combined central and obstructive sleep apnoea utilized the stent as an alternative to nocturnal non-invasive ventilatory support.

Two patients with recurrent chest infections, in whom assisted coughing was difficult, benefited from the stoma stents primarily for tracheal toilet.

One patient following respiratory wean required a further four months of tracheal access on account of episodic hypoventilation and temporarily had a tracheal stent as an inpatient.

Following a questionnaire survey of the patients still living (Appendix 1), all five patients describe less discomfort from the stent system compared with a tracheostomy tube both in day-to-day use and at times of tube change. They felt that the stent was cosmetically superior and the occlusive plug allows for easy vocalization. Following discussion with the specialist nurse practitioners, they also agreed with the patient consensus that stents were easier to clean and maintain and that patient comfort was greater. In addition, they (and we) felt that there were fewer problems associated with the stents compared with tracheostomy tubes, such as dislodgement and stoma infection or other problems that would require acute admission to NWRSIC.

\section{Discussion}

Several different tubes have been developed as alternatives to tracheostomy tubes, although the soft silicone sleep apnoea tube (Bivona) most closely resembles a tracheostomy tube. Other similar devices include the Boston Montgomery longterm cannula ('www.bosmed.com/pdf/trachcat.pdf') and there are numerous cases reported of the use of tracheal T-tubes in cases of tracheal stenosis and injury. ${ }^{2-4}$ These are difficult to maintain clear, free of mucus plugs, in persons who lack active coughing and it is not possible to change the T-tube in the community.

Rather simpler are the tracheal stoma stents and to date, there have been no reports of the use of stoma stents in this clinical situation.

\section{Hood stoma stent system}

The devices are flexible, self-retaining and non-irritant silicone stents (Figure 1). The inner flanges prevent inadvertent ejection of the stent, especially during swallowing.

At the time of insertion, after cleaning the stomal site, these inner flanges are held together with light forceps as the stent is directed through the epithelialized stoma, predominantly without the need for any topical anaesthesia. The outer flanges prevent the stent receding into the trachea (Figure 2).

The Hood stent comes with a detachable plug. For the patient with obstructive sleep breathing, this is removed at

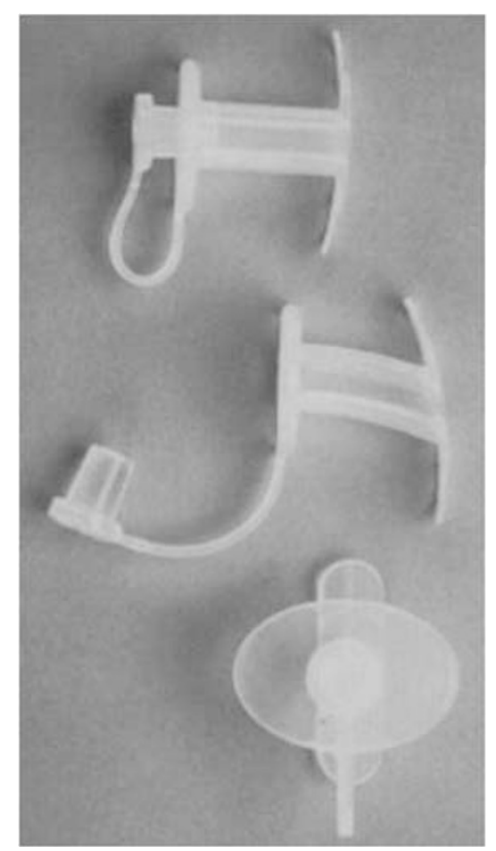

Figure 1 Hood tracheal stoma stent (Reproduced by kind permission of Hoodlabs).
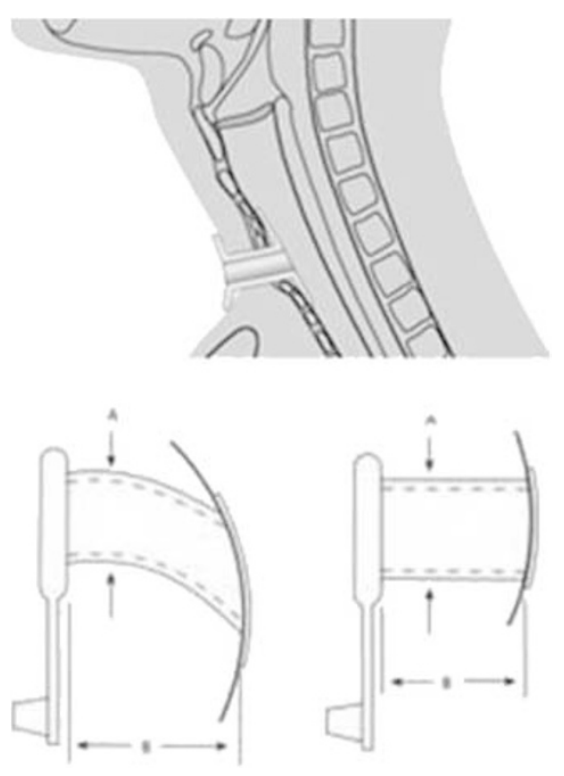

Figure 2 Stent in situ.

night and, using a 15-mm tracheal tube connector, a small heat and moisture exchanger is fitted externally.

Stents are available with external diameters $(\mathrm{mm})$ sizes 8 , 11,13 and 15 and lengths $11-50 \mathrm{~mm}$, and either straight or oblique. Accurate sizing is required to avoid the stent falling out if too short or causing irritation if too long, which may be undertaken using the customized measuring device. The manufacturer's recommendation is that the stents should be changed every day for cleaning with 
sterile water and/or hydrogen peroxide. However, in our practice, monthly changes have proved satisfactory and are cost effective over time compared with regular tracheostomy changes.

\section{Conclusions}

Our experience with stoma stents has been a positive one both for the patients and clinicians alike, and could be used more widely in high tetraplegia. They are more comfortable than a tracheostomy tube and are associated with minimal tracheobronchial secretions. The change of stent is more demanding in comparison with a tracheostomy tube but with staff training can be undertaken in the community on a planned monthly basis.

\section{Conflict of interest}

The authors have no financial considerations to disclose or competing interests in relation to this article.

\section{References}

1 Soni BM. Use of Phrenic nerve stimulator in high ventilator dependent spinal cord injury. Conference proceedings; Network for rehabilitation using functional electrical stimulation for therapy and function restoration 2002. http://fesnet.eng.gla.ac.uk/.

2 Cooper JD, Pearson FG, Patterson TR, Ginsberg RJ, Goldberg M, Waters $P$. Use of silicone stents in the management of airway problems. Ann Thorac Surg 1989; 47: 371-378.

3 Hung-Chang L, Kuo-Sheng L, Charng-Jer H, Ching-Ron C, Wen$\mathrm{Hu} \mathrm{H}$, Ming-Hsiung $\mathrm{H}$. Silicone T-Tube for complex laryngotracheal problems. Eur J Cardiothorac Surg 2002; 21: 326-330.

4 Jer-Huang J. Use of the silicone T-tube to treat tracheal stenosis or tracheal injury. Ann Thorac Cardiovasc Surg 2001; 7: 192-196.

\section{Appendix 1}

\section{Patients' view of the stents}

Was/ls the stent more comfortable than a standard trachesotomy? Is the stent easy to insert?

Are you able to speak adequately with the stent inserted?

Is your speech improved with the stent compared to a tracheostomy tube?

Do you prefer the stent to a standard tracheostomy tube?

Is there any design feature you would change about the stent?

If yes, please give details
Every day*

Every $2-3$ days

Every week

*Delete as appropriate

What size stent do/did you use?

Thank you very much for your time
Every 2-3 weeks

Every month

Every 2-3 months

Every more than 2 months
No 